\title{
Interactive Experiences in Public Spaces: A Novel Floor Display System Based on Luminous Modular Units
}

\author{
Jorge D. Camba \\ University of Houston \\ jdorribo@uh.edu
}

\author{
Jeff Feng \\ University of Houston \\ ffeng@uh.edu
}

\author{
EunSook Kwon \\ University of Houston \\ ekwon@uh.edu
}

\begin{abstract}
We describe the design of an interactive concrete floor display for public spaces and discuss its implementation and areas of application. The primary purpose of our system is to provide new forms of collaborative interaction in long-term installations in both indoor and outdoor spaces. Our design is an expandable system of interconnected tiles, which control an array of RGB Light-Emitting Diodes (LEDs) based on user input. The LEDs are built into the tiles and arranged in a manner that simulates pixels on a computer screen, providing a natural visualization mechanism. Each tile acts as an independent node in a network, where motion sensors trigger specific actions and provide interactivity to the surface. A series of applications are illustrated, including promotional advertising and interactive games. The system was installed and evaluated on a university campus for four weeks where hundreds of visitors successfully interacted with the floor.
\end{abstract}

\section{Introduction}

With the advent of ubiquitous computing, the field of Human-Computer Interaction (HCI) has shifted its focus from interfaces that rely on the user's undivided attention to autonomous systems that connect with the environment and are context-aware [1].

Today, smart sensing and ubiquitous computing technologies are rapidly expanding into everyday products and environments [2]. Given the power and applications of these technologies, researchers have long envisioned small, affordable devices distributed throughout everyday objects and used to connect and enhance our experiences and interactions with these objects [3].

With the availability of low cost and more powerful and reliable sensing technologies, it is now possible to design devices and environments that consistently react to the presence of the user. In addition, when sensor- based controllers are combined with new lighting systems, possibilities emerge for creating responsive interactive display environments. In this context, new LED technologies are gradually transforming the industry and changing the role of lighting beyond merely functional illumination [4]. Large interactive surfaces are becoming commonplace. Responsive floors that provide output to users have applications in numerous areas such as marketing, entertainment, interactive art, and safety and guidance systems.

Although research in the area of interactive floors is not new[5,6], many systems have been designed exclusively for controlled indoor environments or are not prepared to withstand the conditions of long-term outdoor installations. Constant exposure to harsh weather, dust, and heavy pedestrian traffic can severely affect the sensitivity and reliability of these spaces and their components. In public settings where electronic displays, expensive equipment, and top mounted projectors are not suitable, new systems need to be developed that take into consideration the inherent constraints imposed by the environment. An additional problem in outdoor installations is how to cover large areas while providing a sufficiently high resolution.

From an HCI standpoint, the user experience aspect of the technology is fundamental, as the interactive floor needs to not only provide the required functionalities but also support the user's experience throughout use $[7,8]$. These experiences may range from those of enjoyment and enchantment [9], to social challenge [10], and even serendipity [11].

In this paper, we describe the development, implementation, and preliminary testing of Pixl, a programmable floor installation designed to provide collaborative interactive experiences in public environments. Our contribution is framed in the context of design for urban media and provides an expandable low-cost solution for large areas inspired by empirical research in community interaction technologies [12], movement based interaction techniques [13], and digital facades [14].

Although specifically designed for long term installations in outdoor spaces (initial explorations 
focused on a variety of scenarios such as running trails, exercise areas, picnics, entertainment, parks, and green-spaces for community development and urban beautification), Pixl can also be used indoors, even as a vertical surface (if installed on a wall), which can drastically broaden its areas of application.

\section{Related work}

The integration of multimedia displays and information systems into the built environment has received significant attention in various areas such as social gaming [15]), sustainability awareness [16], and artistic expression [17]. Under the umbrella term of urban computing [14] or urban informatics [18] researchers are investigating the particular challenges and potentials that this domain presents.

The majority of displays in public environments are installed vertically to compete with store windows and other signage and are usually integrated in the ecosystem of other visual content, but may easily be overlooked or ignored [19, 20]. Because pedestrians look at the floor frequently [21] and floors are not typically used for displaying information, interactive horizontal displays provide an interesting alternative for developing digital content. For example, one can take advantage of the subtleties in defining areas and accessibilities on floors such as changes in color, material and light [22]. However, while architecture and urban planning have a long tradition of designing floors, incorporating the interactive element to the floor and defining its role in the environment present unique challenges to researchers. Understanding human-computer interaction when computer technology is ubiquitously built into floors is a relatively unexplored topic [22]. According to authors Rodden and Benford [23], much research in ubiquitous computing has focused on "objects" and has not yet explored higher levels of "space," such as floors.

Related work in the area of interactive floors can be classified in two major categories: sensor-based and projection-based systems. Sensor-based interactive floors use sensors to track the position and movement of the user within the surface. Early examples of these floors include the work by Johnstone [5] and Pinkston [6] where electrical contacts and sensors are used to control musical instruments.

In recent developments such as the Magic Carpet [24]), the Robotic Room [25], the interactive floors by Leikas et al. [26], Srinivasan et al. [13], or the Ada floor [27], pressure sensor technology is used to track different aspects of the movement of the user's body such as presence and footstep profiles. Similarly, the Litefoot floor [28] uses input from light sensors to produce and manipulate sound and music. Various multi-user sensor-based interactive floors such as BodyGames [29] have been successfully applied to gaming. More recently, pressure-sensing floors that can interface with context-aware services have been developed [30].

Modular solutions have also been proposed. For example, the interactive Z-tiles developed by Richardson et al. [31] can join together in a variety of ways to form a reconfigurable flexible surface. Each Ztile uses force-sensitive resistors to detect pressure. This information is then used to drive a self-organized network formed by the floor nodes.

Alternatively, interaction with projection-based floors such as iFloor [12] and similar commercial products is based on projection systems and computer vision tracking and gesture recognition technologies. In these systems, the floor space is usually projected from an overhead projector, which allows more fluid visualizations but generally requires installations in precisely controlled environments and carefully calibrated systems. In addition, projector-based computer vision techniques have also difficulties in providing effective multi-user interactions and overcoming occlusion problems [30, 32].

Two creative examples of projection-based floors include GravitySpace [33] and BaseLase [34]. GravitySpace [33] is a back-projected floor prototype designed for indoor spaces where user information is automatically inferred based on the pressure imprints people and objects leave on the floor. BaseLase [34] is an interactive laser floor display suited for large public spaces where user interaction is provided by depth cameras. The prototype described by the authors uses a low-powered laser source and a series of carefully calibrated mirrors that can move to create high resolution focus areas for users. However, as the authors point out, occlusion can become a significant problem in cases of multi-user interaction. In addition, safety may be a concern in practical implementations due to the use of lasers that can damage the user's eyes. A comparison of tactile floors is provided in Table 1.

Some of the challenges involved in designing for urban media were identified by Dalsgaard and Halskov [14]. The most relevant ones (for the purposes of this paper) are described below:

- New interfaces: urban setting prompts new forms of interfaces or alternative assemblies and uses of existing ones

- Integration into physical structures and surroundings: New installations and systems [must be integrated into existing physical surroundings.

- Increased demands for robustness and stability: Shifting light and weather conditions 
Table 1. Comparison of Tactile Floors (N/A: Not Available)

\begin{tabular}{llccccc}
\hline Year & \multicolumn{1}{c}{ Floor } & Area $\left(\mathrm{ft}^{2}\right)$ & \# Tiles & \# Sensors & Scalable & Technology \\
\hline 1991 & Johnstone [5] & N/A & N/A & N/A & N/A & Electrical contacts \\
1994 & Pinkston [6] & 64 & 64 & 64 & N/A & Force sensitive resistors \\
1997 & Paradiso et al. [24] & 60 & N/A & 16 x 32 wires & N/A & Piezoelectric \\
1998 & Fernström \& Griffith [28] & 20 & 121 & 1,936 & N/A & Passive photodetectors \\
2002 & Morishita et al. [25] & 40 & 16 & 65,000 & N/A & Electrical in tactile PCBs \\
2003 & Leikas et al. [26] & N/A & 49 & 196 & N/A & Pressure sensors \\
2004 & Krogh et al. [12] & 215 & N/A & Vision-based & N/A & Ceiling-mounted projection \\
2004 & Richardson et al. [31] & N/A & N/A & $20 /$ tile & Yes & Force sensitive resistors \\
2005 & Lund et al. [29] & 30 & 64 & 64 & Yes & Force sensitive resistors \\
2005 & Srinivasan et al. [13] & 287 & 128 & 258,000 & Yes & Pressure sensors \\
2007 & Delbrück et al. [27] & 1,460 & 360 & 1,080 & Yes & Force sensitive resistors \\
2010 & Chang et al., [30] & 32 & 36 & 144 & Yes & Switch sensors \\
2012 & Karino et al. [32] & 80 & N/A & 1 & Limited & Infrared radar \\
2013 & Bränzel et al. [33] & 86 & N/A & $1+$ camera & N/A & Force sensor+camera \\
2015 & Müller et al. [34] & 800 & N/A & Dept cameras & Limited & Laser projection \\
& Pixl & $\mathbf{1 8}$ & $\mathbf{1 8}$ & $\mathbf{1 4 4}$ & Yes & Passive Infrared sesnsors \\
\hline
\end{tabular}

over which designers often have little or no influence must be taken into account.

- Developing content to suit the medium: The content has to fit the format of the display and the kinds of interaction intended to be supported.

- Aligning stakeholders and balancing interests: Exploring, negotiating, transforming, and balancing stakeholder interests can be critical to the success of a system.

- Transforming social relations: The introduction of new technologies can cause disruptions and transform social relations and protocols.

- Emerging and unforeseen use of places and systems: Media will likely be used, perceived and appropriated in different ways than designers intend.

For the particular case of building interactive floors, the key challenges were listed by authors Delbrück et al. [27]:

- Accurate user detection.

- Physical and electrical robustness, maintainability, and user safety during continuous operation.

- Robust bi-directional communication with a large number of the floor tiles.

- Generation of highly visible, aesthetically pleasing, controllable illumination.

- Scalable and reusable control software infrastructure.

- Engaging and intuitive user interactions.

The authors also state that efforts to date perform well in one or two areas, but not in all of them. The complexity of building such a floor as well as the operational costs and maintainability issues of designing for outdoor settings may explain why there are no permanent instances of interactive floors in realworld, everyday environments [27]. In this regard, our approach provides a robust low-cost solution for these scenarios.

Illuminated floor displays, particularly those based on LED technology, are generally used as secondary spaces, assisting users in interacting with the main display [35]. The floor display can be used to notify users about specific actions or interaction zones with the primary interactive display [35]. More advanced LED-based floor displays use physical tiles as luminous colored elements that can be turned on or off based on user input. Using a computer screen analogy, the entire tile is treated as a single pixel, which makes displaying information difficult due to the low resolution of the overall surface. In addition, most interactive floor systems are designed for controlled indoor environments or as temporary outdoor installations for special exhibits or events.

On the basis of this work, we address practical challenges of urban media, particularly interactive floors, and discuss how we have addressed those challenges in our experimental design. In this paper, we describe the development of a self-contained modular concrete floor designed to work as an interactive display and intended to be used in longterm installations both indoors and outdoors.

Our system is designed for robustness and durability. It implements motion sensor technology to track user actions and a series of RGB LEDs that act as physical pixels on the concrete. Each concrete tile consists of 36 points of light that can be controlled independently, which significantly increases the resolution of the overall display, compared to setups with a single tile as one controlling unit. 


\section{Design}

Our proposed floor is designed as a series of modular tiles that can connect to one another. This modularity allows scalability, i.e., the interactive surface can be made larger or smaller by simply adding or removing tiles. Each tile is a self-contained unit that can function by itself or in combination with other tiles. Furthermore, the spacing between tiles is not constrained, i.e., the tiles can be placed close together or far apart from each other, if necessary, depending on the application and/or the installation site (although in this type of scenario, it would be necessary to properly secure and hide the wires running between tiles). Our initial prototype was implemented as a surface of $6 \times 3$ tiles, covering a total area of approximately $18 \mathrm{ft}^{2}$.

Each tile consists of a one square foot concrete overlay whose top surface is the only element exposed to the environment and the interface between the user and the system. Concrete was selected over other outdoor construction materials such as stone, wood, or glass because of its durability, moldability, and low cost. A gridded internal structure provides support and space for the internal circuitry, and a sheet metal utility box houses the electronic panel and sensors. The utility box is waterproof and intended to be buried in the ground in an outdoor setting. The components of the tile are illustrated in Figure 1. Although usable during the day, light experiences with the proposed interactive floor are naturally richer and more intense at night.

The design of the tile, utility box, and support structure was critical; as the floor would be installed in an outdoor setting where it would have to withstand heavy pedestrian traffic, light-wheeled vehicles (e.g., strollers and wheelchairs), and harsh weather conditions such as high temperatures, rain, and snow.

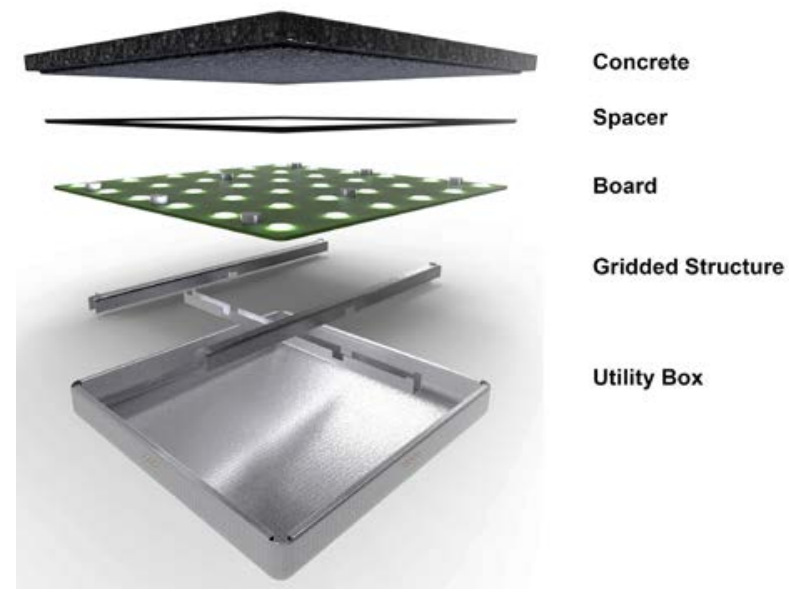

Figure 1. Tile assembly components

\subsection{Physical construction}

The concrete overlay is a 1" thick solid square tile that is casted from custom molds with an embedded steel mesh for reinforced strength. The total weight of the concrete overlay is approximately 10 pounds. More complex shapes can be casted by using other molds, but this could rapidly increase the manufacturing cost of the utility box, which needs to accommodate the tile.

Light is transmitted from the LED panel to the surface via translucent optical fiber clusters that are cast into the concrete. In our implementation, polypropylene plastic rods were selected for prototyping, so passive infrared motion sensors can still be triggered when covered by the rods. The rods varied between two different diameters ( 0.25 inches and 0.125 inches) and are randomly distributed for a mixed more natural visual effect. The plastic clusters are randomly distributed to diffuse the light from the LEDs and physically limit the radius of action of the sensors. For fabrication purposes, we used a low/medium density population of clusters per tile. Higher resolution displays can be produced by increasing the number of clusters and the number of LEDs per board. The surface of the tile can be sanded, textured, or painted, depending on the application and the particular constraints of the installation site. A view of a section of the mold with the polypropylene plastic rods installed is shown in Figure 2. The cast concrete tile with the optical fiber clusters is shown in Figure 3.

The utility box is built from a sheet of steel which is bent to shape with additional pieces welded to form inner structural ribs. Small openings located on both sides allow wires to go through and connect to the subsequent tiles. Power is supplied through the floor to the tiles, which are daisy-chained, allowing power to transfer from one tile to the next. The gridded structure fits inside the box and was designed to keep the wires securely attached and routed so they do not tangle or disconnect during use or installation.

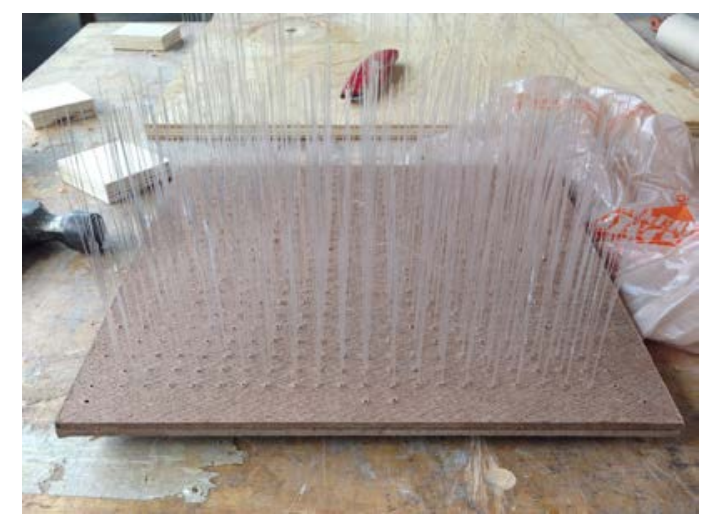

Figure 2. Bottom mold with plastic rods installed 


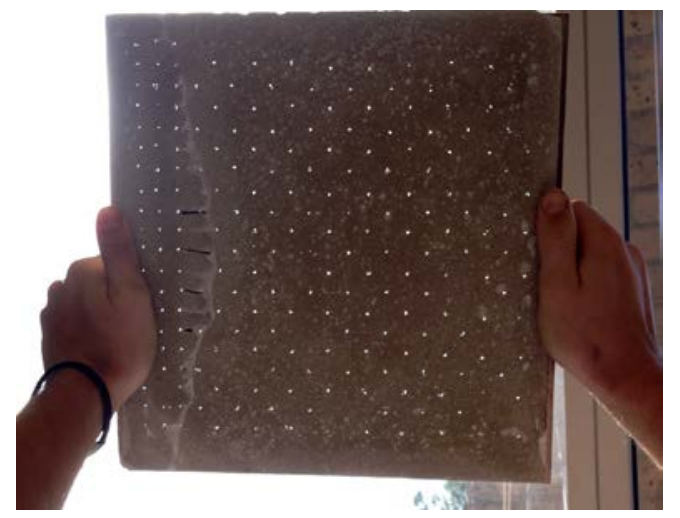

Figure 3. Cast concrete tile

The utility boxes are waterproof, with the openings on the sides sealed. For additional safety, the electronics inside the utility box are mounted on rubber pads, which slightly raise the board from the bottom of the box. Finally, the boxes are designed to be installed as regular tiles secured to the ground, which prevents the tiles from moving. The boxes may be bolted together for extra support. After installation, the space between the concrete tiles can be filled with mortar.

\subsection{Hardware}

A custom circuit board was designed to provide the interactive functionality to the system. The circuit board size matches the concrete tile and is located inside the utility box directly underneath the concrete. It consists of a built-in Arduino Mini microcontroller, 36 RGB LED's (or pixels), 8 passive infrared (PIR) motion sensors, and a communications module that connects the board to the next tile in the system. The approximate peak power use (all LEDs on at maximum brightness) of each tile is $10 \mathrm{~W}$. Lower brightness settings use proportionally less power. PIR motion sensors were selected over other technologies based on considerations of cost, reliability, and performance in the environment.

The RGB LED's are equally distributed on the surface of the board and arranged as a $6 \times 6$ rectangular matrix. The LEDs are based on the WS2812 integrated light source, which uses a built-in driver and a singlewire control protocol to allow each pixel to be uniquely addressed. The light emitted by the LEDs is diffused by the optical fiber rods built into the concrete tile, creating uniform, smooth, and continuous light transitions. Two illuminated tiles with medium density clusters are shown in Figure 4.

To enable a natural real-time user interaction, all sensors in the tile must be read at the same time. This is particularly important in applications where only smaller areas on the tile must be illuminated. Because of limitations in the microcontroller used in our system, only eight input/output pins can be manipulated (read) simultaneously through port registers. Therefore, eight PIR motion sensors are available per tile. The sensors are used to detect when a person has stepped on a specific part of the tile so only the related LEDs light up accordingly. Therefore, for interaction purposes, up to eight contact areas can be detected per tile, depending on the application. The range of the sensors is limited by the optical fiber rods in the concrete tile, so the sensor is triggered only when a user is stepping directly over, instead of when the user is approaching.

All the tiles in our system are connected to a common RS485 bus in a master-slave arrangement, which provides an inexpensive and effective solution for data transmission over relatively large distances, even in electrically noisy environments [36]. The communications module allows the floor space to behave as one system. The schematic of the system architecture is illustrated in Figure 5.

An additional title was installed on the floor and used as a master in our implementation. This title serves as a "selector," so users can cycle through the applications on the display by simply stepping on the master tile. A color scheme is used by the LEDs on the master tile so users can have visual feedback regarding the currently active application.

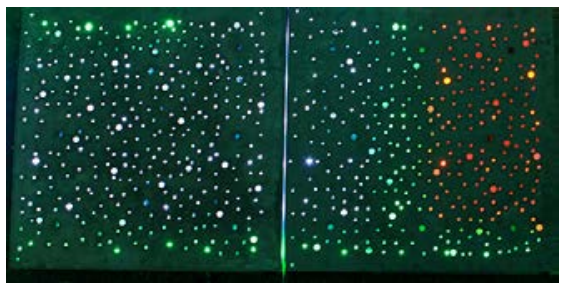

Figure 4. Concrete tiles illuminated with random colors to illustrate cluster density

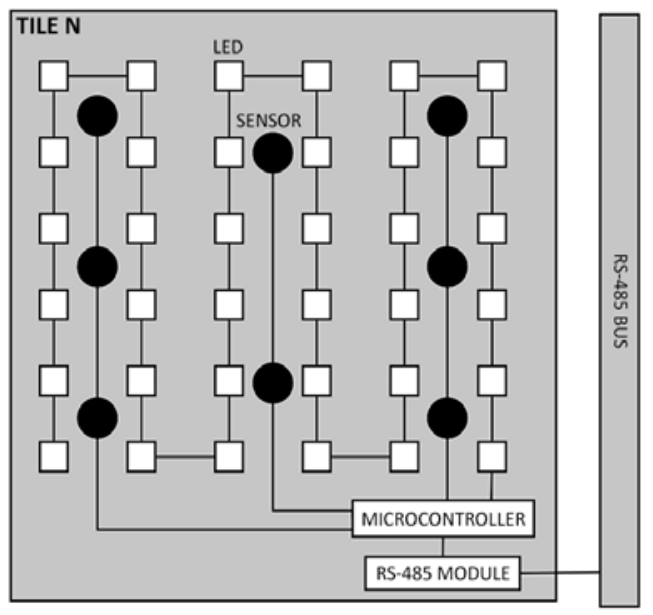

Figure 5. System architecture of tile 


\subsection{Software and Interaction}

The control software for our interactive floor is entirely procedural. Each tile has a microcontroller that regulates the state, color, and brightness of the LEDs and reads data from the motion sensors. The software checks the control tile status and communicates with the floor tiles to select the proper application. Each tile then loops through state specific processing and tile rendering cycles. Because each tile is processed independently by the built-in microcontroller on the board, the overall floor is inherently multi-user. Therefore, traditional 2D image rendering techniques are used where each LED is treated as a pixel on a screen.

We developed algorithms that render static images on the floor (logos, warning signs, messages, etc.) as well as animated visual effects and patterns that can be displayed on certain areas such as pulsating colors and cycle effects. A number of games and experiences to engage users with the system were also developed. Since complex games have been shown to be less effective in engaging the public [27], only simple games or "mini-games" that can be learned in a few seconds were implemented.

The games designed for our interactive floor can be classified as "driven" or "idle" applications. "Driven" programs are light experiences that users can activate themselves. A control tile illuminates and fades slowly and repetitively and serves as a beacon for activation. The user can then engage a program by stepping on the control tile, which will activate a program nearby. Repeatedly stepping on the control tile will toggle through the programs. Examples of these programs include hopscotch, drawing, and pong. "Idle" programs are light experiences that occur automatically and randomly in the public space. These occurrences can serve as a screen saver for the light feature and are more impactful the larger the installation is. They can be designed to run at random times and/or very infrequently, to create a mystique about the public space that can further interest pedestrians. Examples of "idle" applications include the jump meter or the ripple effect.

Games are implemented as a state machine that updates itself on each update cycle. In the "foot trace" game program, for example, the network of LED lights will passively and randomly trace a brief number of strides of the individual as he or she passes over the installation. "Hopscotch" is another application that actively lights up a group of squares to represent a virtual game area. Users are allowed to select the level of difficulty for the game.

\section{Evaluation}

The proposed system is a playful interactive surface intended to engage users and facilitate emotional connections between people and their social and public environments. The installation ran as a public exhibit as part of ID+Green, an industrial design event held at the University of Houston campus (see Figures 6 and 7).

Our initial prototype was notable by its modularity and robustness: the interactive tiles were used for eight hours a day for a period of four weeks. Promotional messages and logos were continuously displayed on the floor and visitors were encouraged to interact with the tiles, which acted as on/off switches. On average, approximately 20 visitors interacted with the system per day. It is estimated that approximately 500 visitors experienced our system throughout the exhibition. Visitors ranged from young children weighing about 40 pounds to adults weighing over 300 pounds. Adults jumping on the tiles can transiently add several hundred pounds. The uptime of the tile electronics was well over 95\%. The only problems found were the occasional loosening of some tiles due to the mortar used to bind the tiles together.

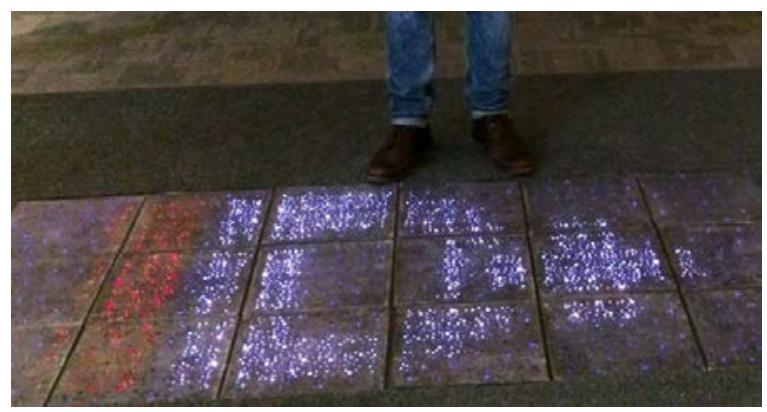

Figure 6. Floor displaying event logo (“ID+”)

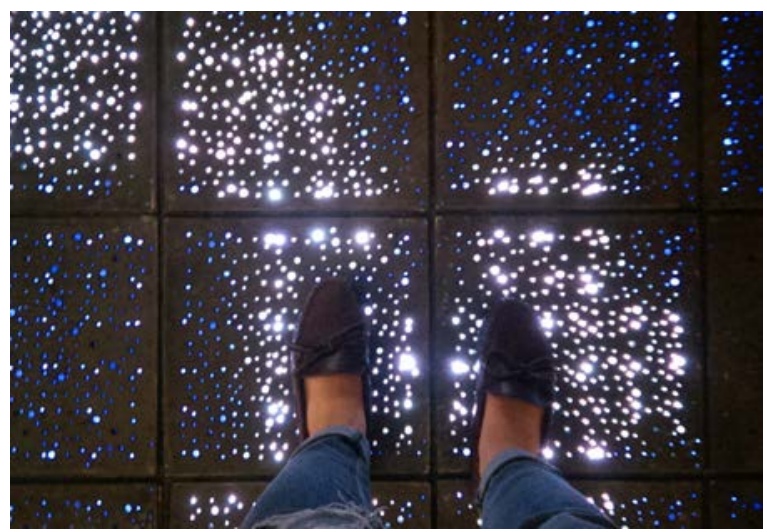

Figure 7. User interacting with floor 
User evaluations were conducted with emphasis on observational studies and semi-structured interviews. Our observations focused on the performance, relevance, and use of the interactive floor, examining the users' reactions and how they experienced the system, and understanding their reflections on how the floor contributed to a traditional public environment.

Our method was to allow users to try the floor and figure out the interaction on their own. The research team would ask a series of questions after the users stepped off the floor. Occasionally, a member of the research team introduced the system to groups of users before allowing them to test it in order to engage in conversations about how the floor was initially perceived. Therefore, information from informal talks conducted before the interaction and semi-structured interviews conducted after the interaction was collected. Questions ranged from factual inquiry, such as how long users interacted with the floor or whether they tried it more than once, to questions regarding usage, communication and understanding.

In terms of system performance, it was interesting to observe how the interactive floor reacted to heavy loads of input. Situations where a large number of participants interacted with the floor at the same time exposed the practical limits of the system. Although in theory the technology could support an unlimited number of users, the fixed number of sensors in the entire surface (144 sensors) and the physical limitations of space (18 square feet) significantly constrain the number of simultaneous users in a practical scenario. In addition, the engaging and social aspects of the interactive experience may be lost when too many users step on the tiles at the same time, as the competitive aspect of certain mini games may be affected by too many inputs.

Occasionally, some participants started using the floor as a medium for personal expression by creating messages on specific areas of the tiles. These interactions were observed once participants identified the location of the sensors on each tile, which provided a more accurate control of the floor display. This result confirms the remarks of Dalsgaard and Halskov [14] which suggest that media will likely be used and perceived beyond its intended purpose [14].

Using the concepts of Dalsgaard \& Hansen [37], the proposed floor can be characterized as a system where the user is simultaneously operator, performer, and spectator. Not surprisingly, direct observations and interviews revealed that many users would watch others interact with the floor before trying it for themselves. As described by Höök et al. [38], the more engaged people are in an activity, the more interesting and appealing it looks for outsiders to try it. Therefore, when designing an unfamiliar space that combines physical and digital elements, it is important to lower the participation barrier and provide users with a possibility to gradually approach the system in a socially safe and welcoming manner.

On average, users spent approximately two to five minutes on the floor. As expected, younger participants used it more often and for longer periods than older adults did.

The general impression and feedback from users was positive. Many interviewees saw the potential of the interactive floor display as a marketing and advertising platform. Furthermore, the floor interface was found both playful and intriguing by many visitors, who commented on the social aspect of the experience and its practicality in a public environment by encouraging people talk to one another. User familiarity and understanding of the floor was noticeable both via body language while interacting with the tiles, and during the conversations and discussions about users' actions and experiences.

Our preliminary study suggests that the text and visuals displayed on our interactive floor are legible and effective in a walk-up and use situation. Our experience and user interviews also confirmed the results reported by Dalton [39], which suggests that pixel density on the display is less important than the visual artifacts introduced by tile edges and that denser LED spacing does not improve legibility or raise user preferences [39]. Although not formally tested, we suspect the same argument applies to the varying diameter rods used on the tiles $(0.25$ inches and 0.125 inches). If true, the cost of manufacturing a tile can be significantly reduced by selecting a single rod diameter for the entire system.

Finally, when asked about their opinion of the concept, the majority of participants interpreted the floor installation either as a large visual information display, an interactive environment, or as a combination of the two. As expected, participants also expressed more interest in the installation at night, when the surroundings are darker and the lights are more predominant.

\section{Conclusions}

We have successfully designed and built the hardware architecture and the control software of a concrete floor system to be used as an interactive display for outdoor environments. Our system was originally designed to increase the value and augment the experience of activities that users perform outdoors while introducing new and engaging activities. This objective was confirmed and validated based on the audience responses and reactions throughout the four- 
week long exhibition. Specifically, our design can incentivize areas of heavy pedestrian traffic. For example, applications such as pedestrian navigation or map-based visualization emerge as particularly relevant. Other fields, including marketing and advertising, entertainment, and collaborative gaming, are also relevant. Because of the modular and ubiquitous nature of the system, indoor spaces can also be enhanced with analogous applications. Additionally, our approach can also be implemented vertically in the form of interactive walls. Examples of application areas are shown in Figure 8.

Our interactive luminous floor offers new forms of interaction in public spaces, and the exhibition where the system was showcased demonstrated that it could be used reliably with the general public. The relatively low cost of the hardware as well as its modularity and low energy consumption make it an attractive solution for large-area installations. As the cost of LED technology continues to decline, higher resolution floors can be developed by incorporating larger

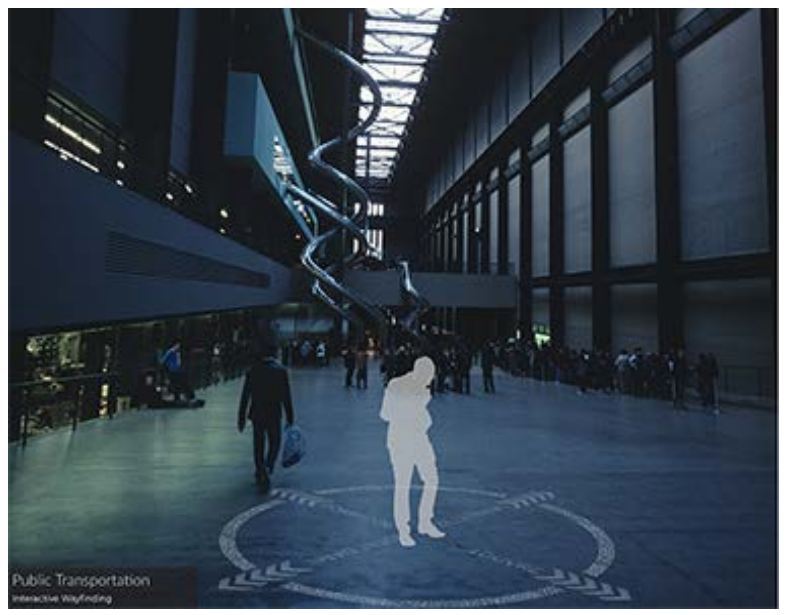

a

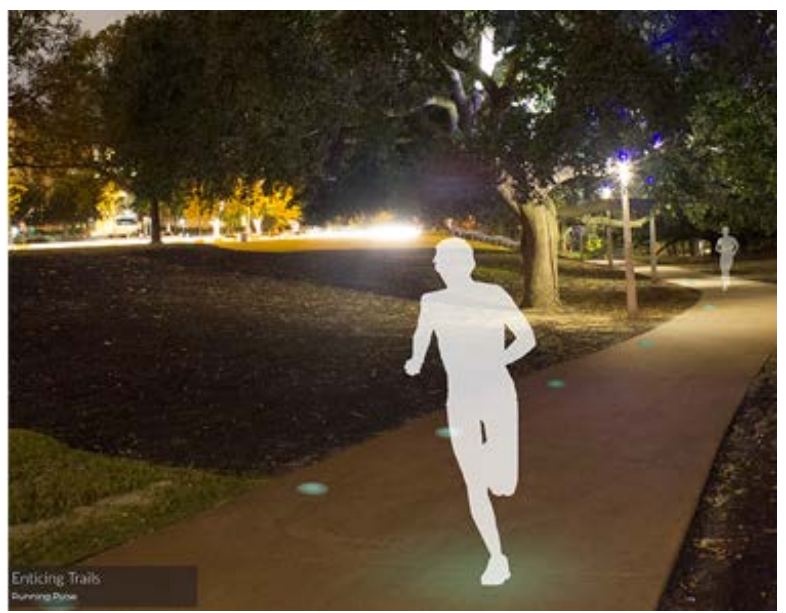

C numbers of LEDs per tile and increasing the density of the optical fiber rods.

In terms of the technical aspects of our design, we noticed that the use of motion sensors needs to be reconsidered. Although the sensitivity of the sensor can be adjusted, it is possible that a user waving her hand over a tile without touching the surface will trigger the sensor. Alternative solutions include distance or ultrasonic sensors, so actions are triggered only when the user's foot is at a certain distance from the tile.

Although still experimental, our interactive floor sheds light on some of the functional and creative possibilities of incorporating interactive technologies in the built environment. In this regard, this type of technology can play a significant role in the fields of architecture, construction, and design. Similarly, more research is needed to determine the most effective integration mechanisms in terms of materials and manufacturing processes, as well as the impact on users, particularly in long-term installations.

As future work, we are interested in developing a

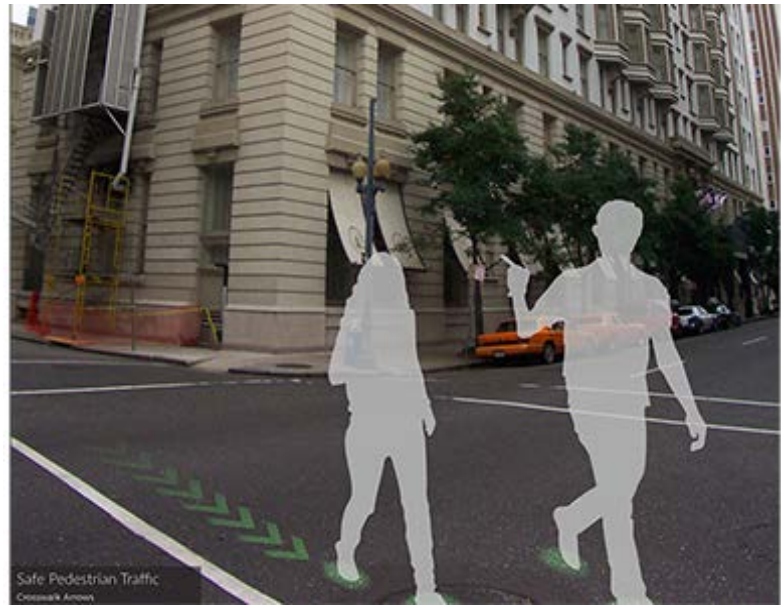

b

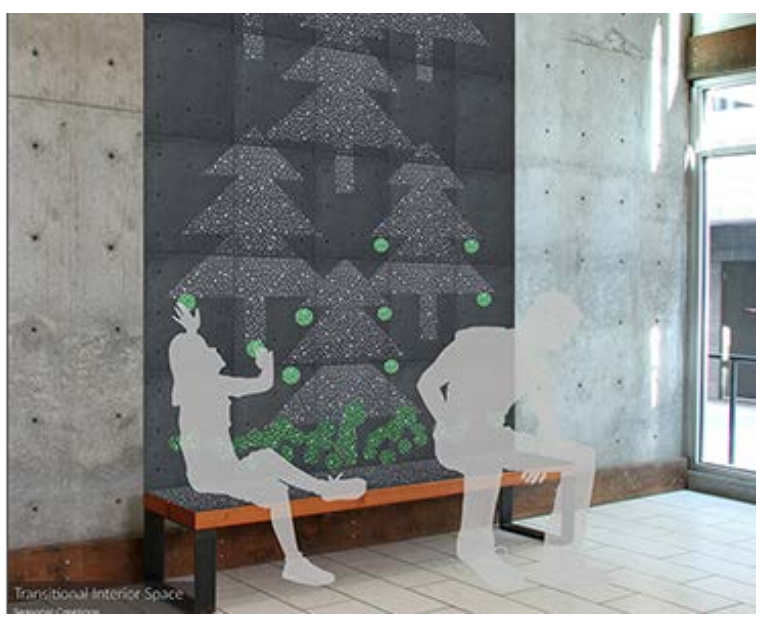

d

Figure 8. Areas of application: (a) navigation, (b and c) user feedback, and (d) marketing and entertainment 
system that can automatically recognize the floor configuration, so new tiles can be added to or removed from the system without having to update the control software manually. This system may take the form of a boot loader that allows the programming of a master tile, which then spreads the program to its neighbors. Additionally, the boot loader can map the physical arrangement of the tiles, thus making the system aware of its own topology, which can facilitate the development of non-rectangular floor configurations and other more complex structures.

The addition of sound to the system and its applicability to new interactive experiences is a shortterm goal whose implementation can be accomplished by slightly modifying the molds of the concrete tile and accommodating a speaker on the circuit board located underneath the tile. There is also room for exploration in terms of the tile construction. For example, new materials, shapes, and sizes as well as different variations and configurations of the plastic clusters can be tested, including diameters, spacing, layout and arrangement. By increasing the number of sensors and LEDs per tile, a more powerful and higher resolution display can be developed.

Finally, we are also interested in expanding the interaction space of our system to other devices. A system where the floor is used as a secondary interaction space may be used to inform users about a specific event or a particular interaction zone, suggest possible actions, and aid users throughout the experience while interacting with a main display.

\section{References}

[1] M. Weiser, "The Computer for the 21st Century", Scientific American, 265, 3, 1991, pp. 94-104.

[2] B.F. Spencer, M.E. Ruiz-Sandoval, and N. Kurata, "Smart sensing technology: opportunities and challenges", Structural Control and Health Monitoring, 11, 4, 2004, pp. 349-368.

[3] S. Poslad, "Ubiquitous Computing: Smart Devices, Environments and Interaction”. Wiley, 2011.

[4] D. Aliakseyeu, B. Meerbeek, J. Mason, A. Lucero, T. Ozcelebi, and H. Pihlajaniemi, "Beyond the switch: explicit and implicit interaction with light”, In: 8th Nordic Conference on Human-Computer Interaction 2014. ACM, New York, NY, pp. 785-788.

[5] E. Johnstone, “A MIDI foot controller - The PodoBoard”, In: Proc. International Computer Music Conference 1991, pp. 123-126.
[6] R.F. Pinkston, "A touch sensitive dance floor/MIDI controller”, Journal of the Acoustical Society of America, 96, 5, 1994, pp. 3302-3302.

[7] J. McCarthy, and P. Wright, “Technology as experience”, Cambridge, Massachusetts: The MIT Press, 2004.

[8] M. Hassenzahl and N. Tractinsky, "User experience - a research agenda", Behaviour and Information Technology, 25(2), 2006, pp. 91-97.

[9] J. McCarthy, P. Wright, J. Wallace, and A. Dearden, “The experience of enchantment in human-computer interaction”, Personal Ubiquitous Computing, 10, 2006, pp. 369-378.

[10] M. Brynskov and M. Ludvigsen, "Mock games: A new genre of pervasive play”, In Proceedings of the 6th Conference on Designing Interactive Systems, 2006, University Park, PA, USA.

[11] T.W. Leong, F. Vetere, and S. Howard, “The serendipity shuffle”, In Proceedings of the 17th conference of the computer-human interaction special interest group (CHISIG) of Australia on Computer-human Interaction (OZCHI), 2005, pp. 1-4.

[12] P.G. Krogh, M. Ludvigsen, and A. Lykke-Olesen, "Help me pull that cursor-A Collaborative Interactive Floor Enhancing Community Interaction”, In: Proc. OZCHI 2004. University of Wollongong, Australia, 2004.

[13] P. Srinivasan, D. Birchfield, G. Qian, and A. Kidané, “A pressure sensing floor for interactive media applications”, In: Proc SIGCHI International Conference on Advances in computer entertainment technology, ACM, 2005, pp. 278281.

[14] P. Dalsgaard and K. Halskov, "Designing urban media facades: cases and challenges”, In: Proc. of CHI'10, ACM, 2010, pp. 2277-2286.

[15] J. Muller, R. Walter, G. Bailly, M. Nischt, and F. Alt, "Looking glass: a field study on noticing interactivity of a shop window”, In: Proc. of CHI’12, ACM, 2012, pp. 297306.

[16] T.W. Leong and M. Brynskov, "CO2nfession: engaging with values through urban conversations", In: Proceedings of the 21st Annual Conference of the Australian ComputerHuman Interaction Special Interest Group: Design: Open 24/7, 2009, pp. 209-216.

[17] L. Bullivant, "Responsive environments: architecture, art and design”, V\&A, London, 2006.

[18] M. Foth, J.H.J. Choi, and C. Satchell, "Urban informatics”, In: Proceedings of the ACM 2011 conference on Computer supported cooperative work, ACM, 2011, pp. 1-8). 
[19] E.M. Huang, A. Koster, and J. Borchers, “Overcoming assumptions and uncovering practices: When does the public really look at public displays?” Pervasive Computing, 2008, pp. 228-243.

[20] J. Muller, D. Wilmsmann, J. Exeler, M. Buzeck, A. Schmidt, T. Jay, and A. Krüger, "Display blindness: The effect of expectations on attention towards digital signage”, Pervasive Computing, 2009, pp. 1-8.

[21] J. Schrammel, E. Mattheiss, S. Döbelt, L. Paletta, A. Almer, and M. Tscheligi, "Attentional behavior of users on the move towards pervasive advertising media”, In Pervasive Advertising, J. Müller, F. Alt, and D. Michelis, Eds., HumanComputer Interaction Series. Springer London, 2011, pp. 287-307.

[22] M.G. Petersen, P.G. Krogh, M. Ludvigsen, and A. Lykke-Olesen, "Floor interaction HCI reaching new ground", In: CHI'05 extended abstracts on Human factors in computing systems, ACM, 2005, pp. 1717-1720.

[23] T. Rodden and S. Benford, "The Evolution of Buildings and Implications for the Design of Ubiquitous Domestic Environments", In: Proceedings of CHI2003. ACM Press, pp. 9-16.

[24] J. Paradiso, C. Abler, K. Hsiao, and M. Reynolds, "The Magic Carpet - Physical Sensing for Immersive Environments”, In: Proc. CHI 97, ACM Press, 1997.

[25] H. Morishita, R. Fukui, and T. Sato, "High resolution pressure sensor distributed floor for future human-robot symbiosis environments", IEEE/RSJ International Conference on Intelligent Robots and Systems, 2, IEEE, 2002, pp. 1246-1251.

[26] J. Leikas, J. Mattila, L. Cluitmans, and T. Urhemaa, "IMS-Intuitive Movement Sensing Method". In Proceedings of the Smart Objects Conference, Grenoble, France, 2003, pp. 200-203.

[27] T. Delbrück, A.M. Whatley, R. Douglas, K. Eng, K. Hepp, and P.F. Verschure, "A tactile luminous floor for an interactive autonomous space”, Robotics and autonomous systems, 55, 6, 2007, pp. 433-443.

[28] M. Fernström and N. Griffith, "Litefoot-Auditory Display of Footwork”. In: Proc of ICAD, 1998.

[29] H.H. Lund, T. Klitbo, and C. Jessen, "Playware Technology for Physically Activating Play”, Artificial life and Robotics Journal, 9, 2005.
[30] S. Chang, S. Ham, S. Kim, D. Suh, and H. Kim, "Ubifloor: design and pilot implementation of an interactive floor system”, IEEE Intelligent Human-Machine Systems and Cybernetics (IHMSC), 2010 2nd International Conference on, Vol. 2, pp. 290-293).

[31] B. Richardson, K. Leydon, M. Fernstrom, and J.A. Paradiso, "Z-Tiles: building blocks for modular, pressuresensing floorspaces”, In: CHI'04 extended abstracts on Human factors in computing systems, ACM, 2004, pp. 15291532.

[32] A. Karino, T. Osugi, Y. Kimura, and T. Takami, "Development of exertainment with interactive floor display”, In: 1st IEEE Global Conference on Consumer Electronics 2012, pp. 99-100.

[33] A. Bränzel, C. Holz, D. Hoffmann, D. Schmidt, M. Knaust, P. Lühne, R. Meusel, S. Richter, and P. Baudisch, "GravitySpace: tracking users and their poses in a smart room using a pressure-sensing floor", In: Proceedings of the SIGCHI Conference on Human Factors in Computing Systems, ACM, 2013, pp. 725-734.

[34] J. Müller, D. Eberle, and C. Schmidt, "BaseLase: An Interactive Focus+ Context Laser Floor”, In: Proceedings of the 33rd Annual ACM Conference on Human Factors in Computing Systems, ACM, 2015, pp. 3869-3878.

[35] J. Vermeulen, K. Luyten, K. Coninx, N. Marquardt, and J. Bird, "Proxemic Flow: Dynamic Peripheral Floor Visualizations for Revealing and Mediating Large Surface Interactions", In: Proc. Human-Computer InteractionINTERACT 2015. Springer International Publishing, 2015, pp. 264-281.

[36] Texas Instruments, "Interface Circuits for TIA/EIA-485 (RS-485)”, Application Report, March 2007.

[37] P. Dalsgaard, and L.K. Hansen, "Performing perceptionstaging aesthetics of interaction", ACM Transactions on Computer-Human Interaction, 15, 3, 2008.

[38] K. Höök, D. Benyon, and A.J. Munro, "Designing information spaces: the social navigation approach", Springer-Verlag London, UK, 2003.

[39] N.S. Dalton, “TapTiles: LED-based floor interaction”, In: Proc. 2013 International Conference on Interactive Tabletops and Surfaces. ACM, 2013, pp. 165-174. 